\title{
Desenvolvimento osteológico de Hippocampus reidi Ginsburg (Pisces, Syngnathiformes, Syngnathidae), em laboratório. I. Período embrionário
}

\section{Rosana Beatriz Silveira ${ }^{1,2}$}

\begin{abstract}
Osteologic Development of Hippocampus reidi Ginsburg (Pisces, Syngnathiformes, Syngnathidae), Under Laboratory Conditions. I. Embryonic Phase. The cartilaginous structures of the embryos of Hippocampus reidi Ginsburg, 1933, were described as well as the beginning of the ossification process in the dentary, angular, retroarticular, operculum, frontal, supraoccipital bones and neural end hemal arches. The viteline sac is well developed. The results show that cartilaginous structures are predominant in specimens with an average of $4.85 \mathrm{~mm}$ in total length.

KEY WORDS. Hippocampus, seahorse, osteology, fishes
\end{abstract}

A família Syngnathidae inclui os cavalos marinhos (Hippocampinae) e peixes-cachimbo (Syngnathinae). São 55 gêneros e aproximadamente 200 espécies. A sistemática dos Syngnathidae é confusa. Somente no gênero Hippocampus, existem 35 espécies, algumas delas necessitando de estudos para confirmar sua validade taxonômica (AZZARELLO 1990). Os cavalos marinhos habitam estuários, mangues, recifes e águas litorâneas nos seis continentes, sendo que no Brasil, encontramos somente duas espécies: Hippocampus reidi Ginsburg, 1933, o cavalo marinho do focinho longo, e $H$. erectus Perry, 1810, o do focinho curto. O primeiro, mais abundante, domina a costa e estuários brasileiros de norte a sul, em relação ao segundo, já tendo sido citado para o estuário da Laguna dos Patos (CHAO et al. 1982).

O estudo do desenvolvimento ósseo em juvenis de peixes tem dado suporte a trabalhos nas mais variadas áreas. A sistemática filogenética (FRITZSCHE 1984; AZZARELLO 1989, 1990), por exemplo, é uma das áreas que se valem muito deste conhecimento, como também a biologia e ecologia (PAUlus 1994). A obra de AzZARELlo (1990) é, até o momento, o único trabalho em Syngnathidae que descreve o desenvolvimento ósseo embrionário até o adulto de espécies desta família.

Nosso estudo descreve o desenvolvimento ósseo de $H$. reidi, em fase inicial, onde geralmente, entre os peixes, existe uma maior sensibilidade a produtos tóxicos solubilizados em água e capazes de afetar a formação do sistema esquelético (RuIVo 1972; MCCANN \& JASPER 1972; HOSE \& HANNAH 1984; SOMASUNDARAM et al. 1984; HENRY 1997). Observações pessoais mostraram-nos ser H. reidi sensível a poluentes ambientais em várias fases de desenvolvimento.

1) Laboratório de Aquicultura Marinha. Avenida Delmar Rocha Barbosa 563, Parque Santa Fé, 91180-490 Porto Alegre, Rio Grande do Sul, Brasil. E-mail: labaquac@yahoo.com

2) Departamento de Zoologia, Universidade Federal do Rio Grande do Sul. Avenida Paulo Gama, prédio 12105, Bom Fim, 90040-600 Porto Alegre, Rio Grande do Sul, Brasil. 
Com os resultados de nossos estudos, pretendemos contribuir para futuros trabalhos sobre morfologia e evolução dentro do grupo, bem como trabalhos aplicados em ecologia e biologia, ressaltando que os cavalos marinhos já estão em risco de extinção pelo grande comércio que envolve países de todo o mundo, inclusive o Brasil (VINCENT 1994a).

\section{MATERIAL E MÉTODOS}

Foram adquiridos, de um pescador de peixes ornamentais marinhos, cinco casais de cavalos marinhos da espécie $H$. reidi para estudos de laboratório. Os espécimes foram provenientes da Barra de São Miguel, Maceió, AL, Brasil.

Utilizou-se para descrição da fase embrionária, embriões originados do aborto de um espécime macho (estresse do transporte), ocorrido à chegada ao laboratório. O material foi imediatamente fixado em formalina $10 \%$ para posterior diafanização. A técnica de diafanização seguiu o método de DINGERKUS \& UHLER (1977). Foram diafanizados 35 espécimes embrionários. Os animais, devidamente corados, foram examinados em microscópio estereoscópio e microscópio biológico com câmara clara acoplada; as fases do desenvolvimento ósseo foram identificadas e registradas por desenhos e fotografias. Os espécimes foram medidos em ocular micrométrica.

A terminologia adotada para os estágios do desenvolvimento em $H$. reidi segue BALON (1975), onde é usado o termo embrião para o período de desenvolvimento dentro do ovo e aqui, ainda, dentro da bolsa incubatória do macho. Salienta-se que os Hippocampinae não possuem estágio larval no ambiente (RYZHKOV 1979) ou seja, esta fase é completamente desenvolvida dentro da bolsa incubatória.

Os embriões examinados apresentaram média de 4,85 $\mathrm{mm}$ (amplitude de 4,6 $-5,0, \mathrm{n}=10)$ de comprimento total $(\mathrm{CT})$, do ápice da cabeça à extremidade da cauda.

A terminologia osteológica segue AzZARELlo (1990).

\section{RESULTADOS}

Partindo da região olfatória, a placa etmóide segue além e dorsalmente ao paresfenóide, finalizando em uma cartilagem na ponta do focinho, chamada cartilagem rostral. Na sua porção distal, a placa etmóide sofre uma curvatura de $180^{\circ}$, para cima e para trás (Fig. 1), dobrando o focinho tubular, e puxando para cima o pré-maxilar e maxilar, ainda em desenvolvimento. Os palatinos, elementos pares colocados de cada lado da cartilagem rostral, tornaram-se muito evidentes em face lateral, quase tocando a extremidade ântero-dorsal do quadrado, passando sobre a placa etmóide encurvada (Fig. 1).

A cartilagem de Meckel é uma estrutura par, muito desenvolvida em relação aos demais ossos da mandíbula. Sob efeito da curvatura da placa etmóide, ela assume posição vertical nesta fase, paralela ao eixo longitudinal do corpo (Fig. 2). Em sua face distal, suas porções convergem e se unem, enquanto que na extremidade anterior, elas divergem e vão conectar-se à porção basal do quadrado. Este tem a porção basal anterior muito alongada, colocando-se sobre o simplético até a cartilagem de Meckel e apresenta sua face superior projetada para dentro da cápsula 


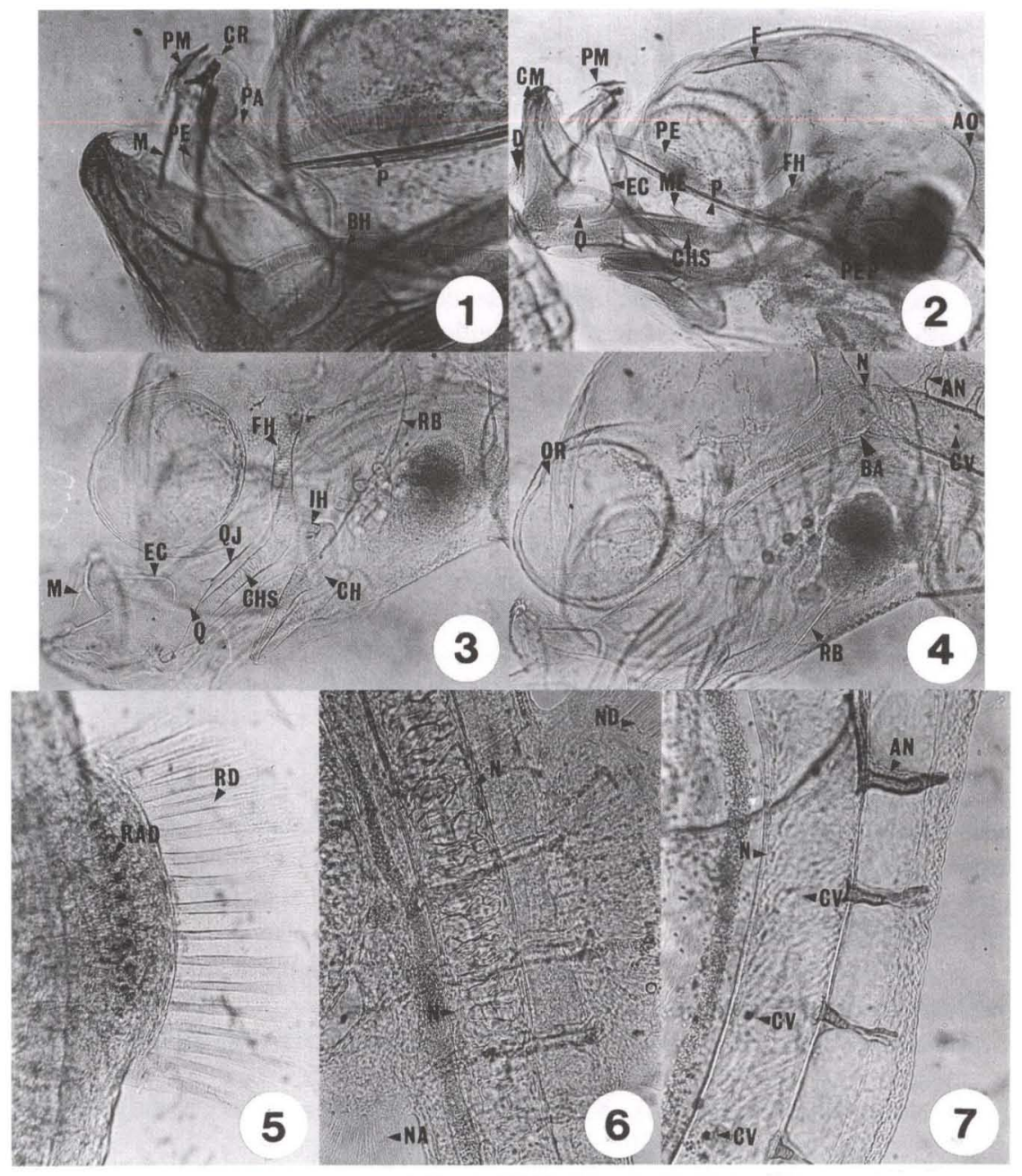

Figs 1-7. Hippocampus reidi, periodo embrionário. (1) Região oromandibular; (2) sincrânio; (3) branquiocrânio látero-ventral; (4) região basicraniana; (5) nadadeira dorsal; (6) região pré-caudal; (7) centros vertebrais em formação. (M) Maxilar, (PE) placa etmóide, (PA) palatino, (PM) pré-maxilar, $(C R)$ cartilagem rostral, $(P)$ paresfenóide, $(B H)$ basi-hial, $(C H S)$ cartilagem hiosimplética, $(\mathrm{Q})$ quadrado, $(\mathrm{D})$ dentário, $(\mathrm{CM})$ cartilagem de Meckel, $(\mathrm{EC})$ ectopterigóide, (ME) metapterigóide, (F) frontal, $(F H)$ forame do hiomandibular; $(A O)$ arco occipital, (PEP) placa esfeno-pterótica, $(\mathrm{QJ})$ quadradojugal, $(\mathrm{CH})$ cerato-hial, $(\mathrm{IH})$ inter-hial, $(\mathrm{RB})$ raio branquiostegal, (OR) órbita, (BA) basioccipital, (N) notocorda, (AN) arco neural, (CV) centro vertebral, (RAD) radial distal, (RD) raio dorsal, (NA) nadadeira anal, (ND) nadadeira dorsal.

óptica (Figs 3, 10). Articulando-se com a cartilagem de Meckel e o quadrado, está o angular, cuja face látero-ventral abriga o retroarticular. O dentário cresce, envolvendo a cartilagem de Meckel (Fig. 2). O quadradojugal é observado, crescendo a partir da face póstero-lateral do quadrado, sobre o simplético, ultrapassando a 
inserção do metapterigóide (Fig. 3). Este último cresce como um acúleo sobre o simplético, em direção à porção superior do quadrado, quase tocando a órbita (Figs $2,10)$. O ectopterigóide situa-se ao longo das faces anteriores do quadrado e palatino (Figs 3, 10). O hiomandibular não apresenta limites visíveis com o simplético, constituindo peça única (cartilagem hiosimplética) e mostra em sua extremidade superior, lateralmente, um forame (Fig. 3). Articulando-se com a face póstero-lateral do hiomandibular está o pré-opérculo, iniciando desenvolvimento, enquanto que o opérculo, já mais desenvolvido, articula-se com a face posterior desta mesma estrutura. $\mathrm{O}$ arco hióide está bem desenvolvido. Observa-se, claramente delimitados, os cerato-hiais, os hipo-hiais, os inter-hiais (pares) e o base-hial (ímpar). O inter-hial é uma pequena estrutura que liga o cerato-hial ao hiomandibular (Fig. 3). Na porção ântero-dorsal do cerato-hial, peça de maior porte do arco hióide, com extremidade posterior bulbosa, liga-se o hipo-hial e da porção anterior deste, parte o base-hial. O basi-hial é uma estrutura cilíndrica, longa e fina que se projeta, anteriormente, para dentro da cavidade oral e posteriormente para a região branquial. Os raios branquiostegais, em número de dois, prendem-se aos cerato-hiais, de cada lado da face (Figs 3,4). O uro-hial, pobremente desenvolvido, é ventral aos cerato-hiais e hipo-hiais e não está em conexão com estes. Os arcos branquiais estão formados, variando em número de três a quatro, dependendo do estágio de desenvolvimento do embrião, e as lamelas branquiais, também, estão formadas.

A nadadeira dorsal exibe entre 16 e17 raios (Fig. 5), 14 ou 15 radiais proximais, dependendo do estágio de desenvolvimento, e 16 radiais distais. Os radiais mesiais não estão bem delimitados, nesta fase. A nadadeira anal possui três raios e três radiais distais, onde num estágio mais avançado evoluem para quatro, tanto os raios, como os radiais. Os radiais proximais da anal, não apresentaram limites bem definidos.

O cleitro é uma fina haste, encurvada, crescendo a partir do primeiro arco neural até a porção basal da nadadeira peitoral. Os radiais peitorais, coracóide e escápula apresentam-se sob forma de placas precursoras, não tomando ainda conformação de estruturas independentes (Fig. 10).

Nesta fase embrionária, ainda não está formada a coluna vertebral. A notocorda (Fig. 6) estende-se da região basicraniana (Fig. 4) até o início da placa hipural, situada no final da cauda. Os centros vertebrais começam a se desenvolver em alguns espécimes (Fig. 7). O atlas projeta-se a partir do primeiro arco neural. Os arcos hemais e neurais, em número de 48 , já estão formados, porém todas as estruturas visualizadas são cartilaginosas e não há sinais de reabsorção deste tecido para deposição de fosfato de cálcio em 31 dos 35 espécimes observados. Em espécimes de desenvolvimento mais avançado, formaram-se as vértebras e está faltando fechar, dorsalmente, o arco neural $($ Figs 8,9$)$ e ventralmente, o arco hemal (Fig. 9). Já estão formados os processos transversos.

Oferecendo suporte estrutural ao crânio, o paresfenóide atravessa-se entre as cápsulas ópticas, indo do basioccipital até a altura do quadrado, ventralmente à placa etmóide (Fig. 10). No neurocrânio, pode-se distinguir o processo pós-orbital como uma estrutura posterior ao olho e uma placa esfeno-pterótica, em vista lateral, que diferenciará em esfenótico e pterótico (Fig. 10). Os frontais desenvolvem-se sobre a porção ântero-dorsal do neurocrânio. Um processo occipital une as duas cápsulas 
óticas. O supra-occipital e o epiótico estão em desenvolvimento (Fig. 10). Ventralmente, visualiza-se o forame magno envolvido pelo exoccipital na região posterior e anteriormente, o pró-ótico.

Quatro dos 35 espécimes observados começam a apresentar sinais de ossificação na região oromandibular e no neurocrânio, os demais apresentavam-se cartilaginos. Observou-se reabsorção de cartilagem e deposição de fosfato de cálcio no dentário, angular, retroarticular, opérculo e frontal, assim como fraca deposição de elementos ósseos nos arcos neurais e hemais e no supra-occipital. As demais estruturas continuam cartilaginosas. O saco vitelínico está bem desenvolvido (Fig. 10).
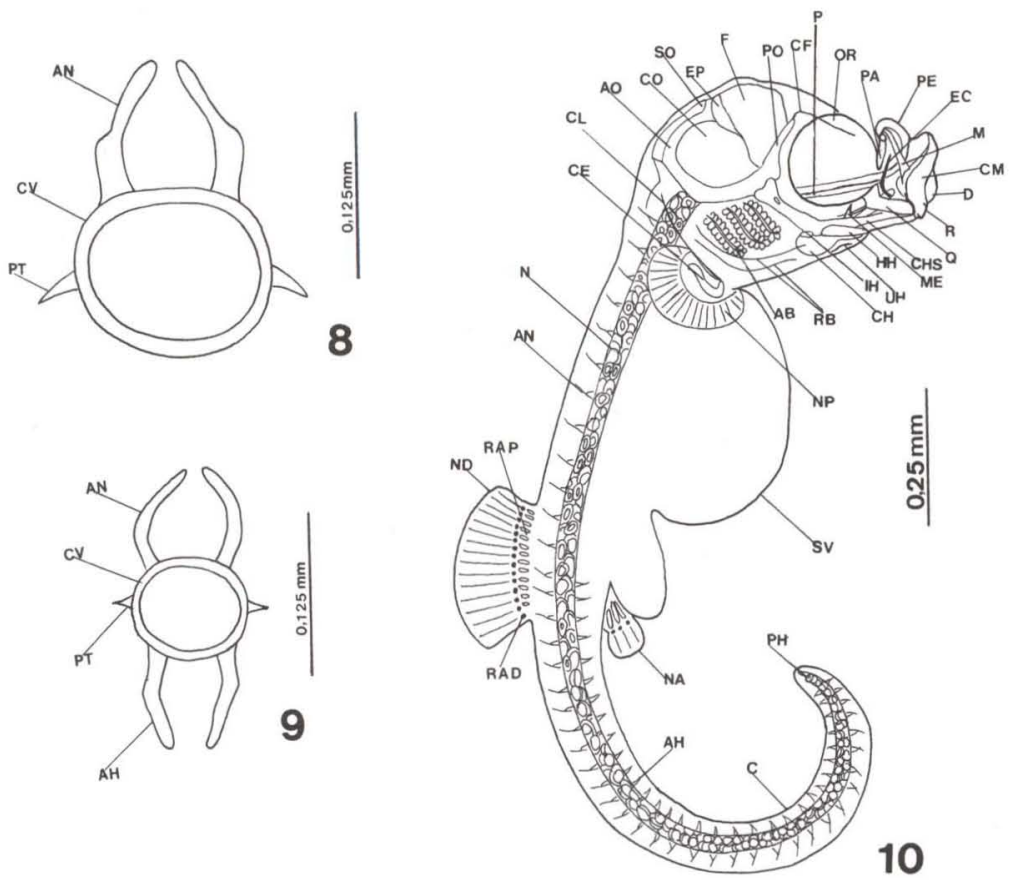

Figs 8-10. Hippocampus reidi, periodo embrionário. (8) Vértebra pré-caudal; (9) vértebra caudal; (10) embrião lateral. (PT) Processo transverso, (CV) centro vertebral, (AN) arco neural, $(\mathrm{AH})$ arco hemal, (PH) placa hipural, $(\mathrm{C})$ cauda, (NA) nadadeira anal, (RAD) radial distal, (ND) nadadeira dorsal, (RAP) radial proximal, $(N)$ notocorda, $(C E)$ coraco-escápula, (CL) cleitro, (AO) arco occipital, (CO) cápsula ótica, (SO) supra-occipital, (EP) epiótico, (F) frontal, (PO) processo pós-orbital, (PA) palatino, (PE) placa etmóide, (EC) ectopterigóide, (M) maxilar, (CM) cartilagem de Meckel, (D) dentário, (R) retroarticular, (Q) quadrado, (CHS) cartilagem hiosimplética, (ME) metapterigóide, $(\mathrm{HH})$ hipo-hial, $(\mathrm{UH})$ huro-hial, $(\mathrm{IH})$ inter-hial, $(\mathrm{CH})$ cerato-hial, (RB) raios branquiostegais, $(\mathrm{AB})$ arcos branquiais, (NP) nadadeira peitoral, (SV) saco vitelinico. 


\section{DISCUSSÃO}

Aos $4,85 \mathrm{~mm}$ de CT médio, o embrião de $H$. reidi exibe caracteres semeIhantes aos descritos por AZZARELLO (1990), para Hippocampus zosterae Jordan \& Gilbert, 1882 e Syngnathus scovelli (Evermann \& kendall), outras duas espécies pertencentes à família Syngnathidae. Durante o período embrionário, a curvatura da placa etmóide para cima e para trás e a rotação da cartilagem de Meckel, tornando-se paralela ao eixo longitudinal do corpo, são vistos como fenômenos adaptados ao desenvolvimento dentro da bolsa incubatória, onde o espaço é limitado (KADAM 1957).

No branquiocrânio do embrião de $H$. reidi, visualiza-se quase todas as estruturas que estarão presentes no jovem, entre estas, o quadradojugal. A presença do quadradojugal é tido como um caráter primitivo, estando presente em crossopterígios e actinopterígios inferiores (WESTOLL 1937; JARVIK 1947). PATTERSON (1973) sugere que, homologamente, a porção posterior do quadrado possa representar o quadradojugal. AZZARELLO (1990) afirma que esta estrutura desenvolve-se a partir de um rudimento parcial ou completamente independente do quadrado em alguns teleósteos, inclusive em $H$. zosterae e $S$. Scovelli. Hippocampus reidi exibe o crescimento do quadradojugal em íntimo contato com o quadrado, porém, como uma estrutura parcialmente independente.

Falta, neste período embrionário, o articular, o endopterigóide e os suborbitais. Para AZZARELlo $(1989,1990)$, estão presentes nas espécies examinadas, todas estas estruturas, porém, o articular não aparecerá no juvenil de $H$. zosterae, somente em S. scovelli. O número de suborbitais diferem entre as espécies de Syngnathidae examinadas por AzZARELLo (1990), H. zosterae apresenta somente o lacrimal e $S$. scovelli, o lacrimal e outro suborbital (não nomeado pela autora). As estruturas presentes e coincidentes nos embriões de $H$. reidi, $H$. zosterae e $S$. scovelli diferem em tempo de ossificação. Para H. zosterae (AzZarello 1990), aos 5,5 mm de comprimento da notocorda $(\mathrm{CN})$, inicia-se o processo de ossificação nos centros vertebrais, pré-maxilar, maxilar, arcos neurais e hemais, dentário, angular, retroarticular, ectopterigóide, uro-hial, paresfenóide, quadradojugal, esfenótico, pró-ótico, basioccipital, exoccipital, cleitro, opérculo e raios branquiostegais. Já para $H$. reidi, ossificação inicia aos 5,0 mm de comprimento total (CT), ou seja, um pouco antes de $H$. zosterae e em menor número de estruturas: dentário, angular, retroarticular, frontal, supra-occipital e arcos neurais e hemais. Para AzZARELLO (1990), a ossificação em $S$. scovelli inicia aos $5,5 \mathrm{~mm}$ de $\mathrm{CN}$, e somente o pré-maxilar apresenta-se com depósito de fosfato da cálcio. Estes princípios de ossificação estão de acordo com WEISEL (1967), o qual afirma que as regiões expostas a um alto grau de estresse, são as primeiras que ossificam nos peixes, como por exemplo, as maxilas, responsáveis pela alimentação e os ossos branquiais que proporcionam suporte e proteção aos órgãos respiratórios. A diferença no tempo de ossificação e número de estruturas a ossificar entre os embriões de $H$. reidi e $H$. zosterae, deve-se, possivelmente, a diferentes estratégias para manutenção das espécies. Hippocampus zosterae $(40 \mathrm{~mm}$ de CT máximo observado) possui uma expectativa de vida de aproximadamente um ano e o macho abriga em sua bolsa incubadora até 50 embriões (VINCENT 1994b). 
Hippocampus reidi (180 mm CT máximo observado) possui expectativa de vida em torno de quatro anos e o macho desenvolve em sua bolsa entre 200 e 1000 embriões (Silveira no prelo). O fato de ter um ciclo de vida menor e prole constituída de poucos espécimes, explicaria a ossificação inicial em um maior número de estruturas, em $H$. zosterae, pela necessidade de um rápido desenvolvimento. Por conter poucos embriões desenvolvendo dentro da bolsa incubadora, $H$. zosterae disponibilizaria um maior conteúdo de cálcio, presente no fluído da bolsa incubadora (LINTON \& SOLOFF 1964), para a ossificação, levando também à um desenvolvimento mais rápido.

De acordo com HARDY (1980), o embrião de H. erectus, aos 3,6 mm de CT, apresenta o corpo pigmentado de pardo, com olhos pretos, saco vitelínico bastante desenvolvido e esférico, nenhuma nadadeira está formada, somente rudimentos e 0 focinho ainda não possui o formato tubular presente no juvenil e no adulto. Aos 4,8 $\mathrm{mm}$ de CT, a espécie acima citada, já possui todas as nadadeiras, o corpo com pigmentação mais forte, o focinho já está alongado na forma de tubo. Igualmente, para o embrião de $H$. japonicus, segundo FrITZCHE (1984), aos 6 mm de CT são exibidas estas características. Nos embriões de Syngnathus fuscos Günther, 1870, aos $3,5 \mathrm{~mm}$ de CT, são mostrados os rudimentos das nadadeiras, inclusive a caudal (que não existe em Hippocampus) e o saco vitelínico é esférico. $\mathrm{O}$ embrião de $H$. zosterae aos 4,5 $\mathrm{mm}$ de $\mathrm{CN}$, apresenta as nadadeiras peitorais desenvolvidas com 20 raios, a dorsal com dez raios e a nadadeira anal forma-se aos $5,5 \mathrm{~mm}$ de $\mathrm{CN}$, com três raios. Para o embrião de $H$. reidi $(4,85 \mathrm{~mm}$ de CT), as nadadeiras peitorais apresentam-se desenvolvidas com 15 raios, a dorsal varia entre 16 e 17 raios e a nadadeira anal com três raios. O saco vitelínico é oval.

Nesta fase embrionária, a coluna vertebral de $H$. reidi ainda não está formada. A notocorda dá o sustento ao corpo, apresentando-se, caracteristicamente, composta por grandes células túrgidas. Em espécimes de desenvolvimento avançado, começa a formação dos centros vertebrais. Para $H$. zosterae, os centros vertebrais aparecem formados aos 4,5 $\mathrm{mm}$ de $\mathrm{CN}$ e aos 5,5 $\mathrm{mm}$ começam a ossificar, enquanto que para $S$. scovelli, os centros vertebrais formam-se aos $8 \mathrm{~mm}$ de CN e ossificam aos 8,5 mm (AZZARELLO 1990).

No neurocrânio de $H$. zosterae, o paresfenóide aparece aos $5,5 \mathrm{~mm}$ de $\mathrm{CN}$ em processo de ossificação, em $S$. scovelli, somente aos 7,5 $\mathrm{mm}$ de $\mathrm{CN}$ e, também, em processo de ossificação (AZZARELLo 1990), para $H$. reidi, esta estrutura faz-se presente aos 4,85 mm, apresentando-se cartilaginosa. A cápsula ótica em $H$. reidi é bastante evidente e o processo pós-orbital apresenta-se como uma cartilagem posterior ao olho que sofrerá ossificação. Da mesma maneira ocorre em $S$. scovelli; entretanto, para $H$. zosterae, o processo pós-orbital não ossifica. A placa cartilaginosa esfeno-pterótica, na face lateral do crânio, inicia ossificação no embrião de $H$. zosterae e $S$. scovelli (AZZARELLO 1990), porém em H. reidi a ossificação tem início somente no período juvenil. Os frontais de $H$. reidi estão em desenvolvimento, fechando a região anterior do crânio e apresentam os primeiros sinais de ossificação aos 4,85 mm de CT. Em $H$. zosterae a ossificação inicia-se aos 7,0 $\mathrm{mm}$ e para $S$. scovelli aos 8,5 mm de CN (AZZARELLO 1990). O supra-occipital começa a ossificar em H. zosterae aos $8 \mathrm{~mm}$, em S. scovelli aos $9 \mathrm{~mm}$ de CN (AZZARELLo 1990) e em 
H. reidi aos 4,85 mm de CT. A taenia tectum medialis é uma cartilagem que está situada entre o supra-occipital e os frontais, aparecendo no embrião de $S$. scovelli (AZZARELlo 1990) e $H$. antiquorum (KADAM 1957) aos 6,5 e 9,0 mm de CN, respectivamente, enquanto que em $H$. zosterae (AZZARELlo 1990) e $H$. reidi aparecerá somente na fase juvenil. Ventralmente, no neurocrânio de $H$. reidi aos $4,85 \mathrm{~mm}$ de CT, permanecem cartilaginosos o exoccipital que envolve o forame metótico e o pró-ótico. Em $S$. scovelli aos $5,5 \mathrm{~mm}$ de $\mathrm{CN}$, tais estruturas também não ossificaram. Já em $H$. zosterae, aos $5,5 \mathrm{~mm}$ de $\mathrm{CN}$, o processo de ossificação tem início (AZZARELLO 1990).

AGRADECIMENTOS. Nossos mais sinceros agradecimentos ao Dr. Luiz Roberto Malabarba, à Dr. Clarice B. Fialho e à Dr. Aline Barcellos pela leitura crítica do manuscrito e demais sugestões na composição do trabalho. À Profa Leda Francisca Armani Jardim pelas sugestões nas análises osteológicas e à CAPES pela bolsa de mestrado.

\section{REFERÊNCIAS BIBLIOGRÁFICAS}

Azzarello, M.Y. 1989. The pterygoid series in Hippocampus zosterae and Syngnathus scovelli. Copeia 3: 621-628.

. 1990. A comparative study of the developmental osteology of Syngnathus scovelli and Hippocampus zosterae (Pisces, Syngnathidae) and it's phylogenetic implications. Evol. Monogr. 12: $1-90$.

Balon, E.K. 1975. Terminology of intervals in fish development. Jour. Fish. Res. Bd. Can. 32: 1663-1670.

ChaO, N.L.; L.E. Pereira; J.P. Vieira Sobrinho; M.A. Bemvenuti \& L.P.R. Cunha. 1982. Relação preliminar dos peixes estuarinos e marinhos da Lagoa dos Patos e região costeira adjacente, Rio Grande do Sul, Brasil. Atlântica 5: 67-75.

DINGERKUS, G. \& L.D. UHLER. 1977. Enzime clearing of alcian blue stained whole small vertebrates for demonstration of cartilage. Stain Technol. 52: 229-232.

FritzSCHE, R.A. 1984. Gasterosteiformes: development and relationships, p. 398-405. In: Symposium on the Ontogeny and Sistematics of Fishes [1983]. La Jolla, American Society of Ichthyologists and Herpetologists.

HARDY JR., J.D. 1980. Development of fishes of the mid-Atlantic Bight, an atlas of egg, larval and juvenile stages. V. II. Anguillidae trhough Syngnathidae. Washington, D.C., U.S. Depart. Interior, $459 \mathrm{p}$.

HENRY, T.R. 1997. Early life stage toxicity of 2, 3, 7, 8-tetrachlorodibenzeno-p-dioxin in zebrafish (Danio rerio). Toxicol. Appl. Pharmacol. 142 (1): 56-68.

Hose, J.E. \& J.B. HanNah. 1984. Histologic and skeletal abnormalities in Benzo(a) pyrene-treated Rainbow Trout alevins. Arch. Environ. Contam. Toxicol. 13: 675-684.

JARVIK, E. 1947. Notes on the pit-lines and dermal bones of the head in Polypterus. Zoologiska Bidrag Fran Uppsala 25: 60-78.

KADAM, K.M. 1957. The development of the chondrocranium in the sea-horse, Hippocampus (Lophobranchii). Jour. Linn. Zool. 43: 557-573.

LinTON, J.R. \& B.L. SolofF. 1964. The phisiology of the brood pouch of the male seahorse Hippocampus erectus. Bull. Mar. Sci. Gulf. Carib. 14 (1): 45-61.

MCCANN, J.A. \& R.L. JASPER. 1972. Vertebral damage to bluegills exposed to acutely toxic levels of pesticides. Trans. Amer. Fish. Soc. 2: 317-322.

PATTERSON, C. 1973. Interrelationships of holosteans. In: Interrelationship of Fishes. Zool. Jour. Lin. Soc. 53 (Suppl. 1): 233-305. 
Paulus, T. 1994. Morphologie des Kopfes und der Röhrenschnause verschiedener Syngnathidae aus dem Roten Meer (Pisces: Teleostei). Senckenbergiana Maritima 25 (1/3): 53-62.

Ruivo, N. 1972. Marine Pollutions and Sea Life. London, FAO, Fishing News Books Ltda., 624p.

RYZHKov, L.P. 1979. Influence of temperature on morphology and physiology of salmonid embryos, larvae and alevins. Pol. Arch. Hydrobiol. 26: 397-425.

SiLVEIRA, R.B. (no prelo). Comportamento reprodutivo e desenvolvimento inicial do cavalo marinho Hippocampus reidi Ginsburg (Pisces, Syngnathiformes, Syngnathidae) em laboratório. Biociências $8(1)$.

Somasundaram, B.; P.E. King \& S.E. ShackLEy. 1984. Some morphological effects of zinc upon the yolk-sac larvae of Clupea harengus L. Jour. Fish. Biol. 25: 333-343.

VINCENT, A. 1994a. The improbable sea horse. Nat. Geogr. 186 (4): 128-140. . 1994b. Operational Sex Ratios in Seahorses. Behaviour 128 (1-2): 153-167.

WEISEL, G.F. 1967. Early ossification in the skeleton of the sucker (Catostomus macrocheilus) and the guppy (Poecilia reticulata). Jour. Morphol. 121: 1-18.

WESTOLL, T.S. 1937. On the cheeck -bones in teleostome fishes. Jour. Anat. 72: 362-382.

Recebido em 27.V.1999; aceito em 11.V.2000. 